\title{
ALGEBRAIC CONVERGENCE THEOREMS OF COMPLEX KLEINIAN GROUPS
}

\author{
WENSHENG CAO* \\ School of Mathematics and Computational Science, Wuyi University, \\ Jiangmen, Guangdong 529020, P.R. China \\ e-mail:wenscao@yahoo.com.cn
}

(Received 7 April 2009; revised 18 April 2012; accepted 4 May 2012; first published online 2 August 2012)

\begin{abstract}
Let $\left\{G_{r, i}\right\}$ be a sequence of r-generator subgroups of $U(1, n ; \mathbb{C})$ and $G_{r}$ be its algebraic limit group. In this paper, two algebraic convergence theorems concerning $\left\{G_{r, i}\right\}$ and $G_{r}$ are obtained. Our results are generalisations of their counterparts in the n-dimensional sense-preserving Möbius group.
\end{abstract}

2000 Mathematics Subject Classification. 30F40, $20 \mathrm{H} 10$.

1. Introduction. Let $\mathbb{G}$ be the $n$-dimensional sense-preserving Möbius group $M\left(\overline{\mathbb{R}}^{n}\right)$ or the unitary group $U(1, n ; \mathbb{C})$.

DEFINITION 1.1. Let $\left\{G_{r, i}\right\}$ be a sequence of subgroups in group $\mathbb{G}$ and each be generated by $g_{1, i}, g_{2, i}, \ldots, g_{r, i}$, where $r=1,2, \ldots$ If for each $t(1 \leq t \leq r)$,

$$
g_{t, i} \rightarrow g_{t} \in \mathbb{G} \text { as } i \rightarrow \infty,
$$

then we say that $\left\{G_{r, i}\right\}$ algebraically converges to $G_{r}=\left\langle g_{1}, g_{2}, \ldots, g_{r}\right\rangle$.

If for each $i, G_{r, i}$ is a Kleinian group, the problem that when $G_{r}$ is still a Kleinian group has been investigated by a number of authors.

When $n=2$, Jørgensen and Klein [7] proved the following.

THEOREM JK. If each $G_{r, i}$ is a r-generator Kleinian group, then the limit group $G_{r}$ is also a Kleinian group.

Examples in [12] show that the Theorem JK could not be extended to $n$ dimensional cases $(n \geq 3)$ without any modifications. The reason for this phenomenon is that there is a great difference in the fixed point set of elliptic elements between $M\left(\overline{\mathbb{R}}^{2}\right)$ and $M\left(\overline{\mathbb{R}}^{n}\right)$ when $n \geq 3$. Several authors have obtained their analogues in $M\left(\overline{\mathbb{R}}^{n}\right)$ when $n \geq 3$ by adding some condition(s) to control the fixed point set of elliptic elements.

Apanasov [1] proved the following.

THEOREM A. If for each $G_{r, i}$, its generators are of infinite order and $G_{r, i}$ is discrete, then for each $t(1 \leq t \leq r), g_{t}=\lim _{i \rightarrow \infty} g_{t, i}$ is different from the identity. Furthermore, if each $G_{r, i}$ is a torsion-free Kleinian group, then $G_{r}$ is also a torsion-free Kleinian group.

Martin [9] proved this.

*Supported by NSFs of China $(11071063,10801107)$ and NSF of Guangdong Province (S2011010000735). 
THEOREM M. Let $G_{r}$ be an algebraic limit group of a sequence of $r$-generator Kleinian groups of $M\left(\overline{\mathbb{R}}^{n}\right)$ of uniformly bounded torsion. Then $G_{r}$ is a Kleinian group.

Wang [11] proved this.

THEOREM W. Let $r<\infty$ and $G_{r}$ be the algebraic limit group of a sequence of $r$ generator Kleinian groups $\left\{G_{r, i}\right\}$ of $M\left(\mathbb{\mathbb { R }}^{n}\right)$. If $\left\{G_{r, i}\right\}$ satisfies EP-condition, then $G_{r}$ is a Kleinian group.

See details in [11] for the definitions of uniformly bounded torsion, EP-condition and $W Y\left(G_{r}\right)$.

A complex hyperbolic space is more complicated than a real hyperbolic space. For example, it has variable negative curvature, it is a Kähler manifold with biholomorphic automorphisms and its boundary has a natural contact structure, which is locally modelled on the Heisenberg geometry. Because of a closed connection between real and complex hyperbolic geometry, the road map of analogy frequently points the way towards potentially interesting questions. It is interesting to investigate analogous results of a real hyperbolic space in the setting of a complex hyperbolic space.

The purpose of this paper is to find analogous results mentioned above in the setting of a complex hyperbolic space. In order to state our main results, we first recall some notations and facts about a complex hyperbolic space.

The complex hyperbolic n-space $H_{\mathbb{C}}^{n}$ may be identified with a unit ball in $\mathbb{C}^{n}$ with the Bergman metric $[\mathbf{6 , 8}, \mathbf{8}$. The group of its holomorphic isometries is the group $U(1, n ; \mathbb{C})$ acting on $H_{\mathbb{C}}^{n}$ and on its boundary $\partial H_{\mathbb{C}}^{n}$. For a non-trivial element $g$ of $U(1, n ; \mathbb{C})$, we say that $g$ is parabolic if it has exactly one fixed point and this lies on $\partial H_{\mathbb{C}}^{n} ; g$ is loxodromic if it has exactly two fixed points and they lie on $\partial H_{\mathbb{C}}^{n}$ and $g$ is elliptic if it has a fixed point in $H_{\mathbb{C}}^{n}$.

For elliptic element $g$, let $\Lambda_{0}$ and $\Lambda_{i}, i=1,2, \ldots, n$ be its negative and positive classes of eigenvalues, respectively. Then the fixed point set of $g$ in $H_{\mathbb{C}}^{n}$ contains only one fixed point if $\Lambda_{0} \neq \Lambda_{i}, i=1,2, \ldots, n$ and is a totally geodesic sub-manifold, which is equivalent to $H_{\mathbb{C}}^{m}$ (for some $m \leq n$ ) if $\Lambda_{0}$ coincides with exactly $m$ of class $\Lambda_{i}, i=1,2, \ldots, n$. We call an elliptic element $g$ an irrational rotation if $e^{\mathbf{i} \theta} \in \Lambda_{t}$ with irrational $\theta$ for some $t$. We remark that $U(1, n ; \mathbb{C})$ has an elliptic element, which has no fixed point in the boundary $\partial H_{\mathbb{C}}^{n}$. Such elements are the counterparts of fixed-point-free elements in $M\left(\overline{\mathbb{R}}^{n}\right)$.

For subgroup $G \subset U(1, n ; \mathbb{C})$, the limit set $L(G)$ of $G$ is defined as

$$
L(G)=\overline{G(p)} \cap \partial H_{\mathbb{C}}^{n}, \quad p \in H_{\mathbb{C}}^{n}
$$

As in [3], for subgroup $G$ of $U(1, n ; \mathbb{C})$ containing a loxodromic element, let

$$
W(G)=\cap_{f \in h(G)} G_{f i x(f)},
$$

where $h(G)$ is a set of all loxodromic elements in $G$ and $G_{f i x(f)}=\{g \in G: f i x(f) \subset$ $f i x(g)\}$.

A subgroup $G$ of $U(1, n ; \mathbb{C})$ is called non-elementary if it contains two non-elliptic elements of infinite order with distinct fixed points that are not irrational rotations; otherwise $G$ is called elementary. We call a non-elementary and discrete subgroup $G$ of $U(1, n ; \mathbb{C})$ a complex Kleinian group. 


\section{ALGEBRAIC CONVERGENCE THEOREMS OF COMPLEX KLEINIAN GROUPS 3}

As in [11], a subset $H$ of $U(1, n ; \mathbb{C})$ is said to have uniformly bounded torsion if there exists an integer $M$ such that

$$
\operatorname{ord}(g) \leq M \text { or } \operatorname{ord}(g)=\infty \text { if } g \in H .
$$

We refer to $[\mathbf{4 - 6 , 8}$ ] for more details of these concepts and some properties of a complex hyperbolic space.

When $\mathbb{G}=U(1, n ; \mathbb{C})$ in Definition 1.1 , we assume that $r<\infty$, and for $\left\{G_{r, i}\right\}$ we introduce the following conditions:

We say that $\left\{G_{r, i}\right\}$ satisfies I-condition if any sequence $\left\{f_{i_{k}}\right\}\left(f_{i_{k}} \in G_{r, i_{k}}\right)$ satisfying that for each $k$, card $\left[f i x\left(f_{i_{k}}\right)\right]=\infty$ and $f_{i_{k}} \rightarrow$ the identity as $k \rightarrow \infty$, has uniformly bounded torsion. Here card $(M)$ denotes the cardinality of set $M$.

We say that $\left\{G_{r, i}\right\}$ satisfies IP-condition if $\left\{G_{r, i}\right\}$ satisfies the following conditions: for any sequence $\left\{f_{i_{k}}\right\}\left(f_{i_{k}} \in G_{r, i_{k}}\right)$, if card $\left(f i x\left(f_{i_{k}}\right)\right)=\infty$ for each $k$, and $f_{i_{k}} \rightarrow f$ as $k \rightarrow \infty$ with $f$ being the identity or parabolic, then $\left\{f_{i_{k}}\right\}$ has uniformly bounded torsion.

As should be apparent, our exposition and results here owe a great deal to Martin's [9] and Wang's papers [11]. Our main results are the following theorems.

THEOREM 1.1. Let $\left\{G_{r, i}\right\}$ be a sequence of groups of $U(1, n ; \mathbb{C})$. If each $G_{r, i}$ is discrete, then the algebraic limit group $G_{r}$ of $\left\{G_{r, i}\right\}$ is either a complex Kleinian group, or it is elementary, or $W\left(G_{r}\right)$ is not finite.

THEOREM 1.2. Let $G_{r}$ be the algebraic limit group of complex Kleinian groups $\left\{G_{r, i}\right\}$ of $U(1, n ; \mathbb{C})$. If $\left\{G_{r, i}\right\}$ satisfies IP-condition, then $G_{r}$ is a complex Kleinian group.

2. Several lemmas. The following lemma is crucial for us.

Lemma 2.1. (cf. [5]). Suppose that $f$ and $g \in U(1, n ; \mathbb{C})$ generate a discrete and non-elementary group. Then

(1) if $f$ is parabolic or loxodromic, we have

$$
\max \{N(f), N([f, g])\} \geq 2-\sqrt{3},
$$

where $[f, g]=f g f^{-1} g^{-1}$ is a commutator of $f$ and $g, N(f)=\left\|f-I_{n+1}\right\|$ and $\|$.$\| is the$ Hilbert-Schmidt norm;

(2) iff is elliptic, we have

$$
\max \left\{N(f), N\left(\left[f, g^{i}\right]\right) \mid i=1,2, \ldots, n+1\right\} \geq 2-\sqrt{3} .
$$

The following lemma is a classification of elementary subgroups of $U(1, n ; \mathbb{C})$.

LEMMA 2.2. (cf. [2]).

(1) If $G$ contains a parabolic element but no loxodromic element, then $G$ is elementary if and only if it fixes a point in $\partial H_{\mathbb{C}}^{n}$;

(2) If $G$ contains a loxodromic element, then $G$ is elementary if and only if it fixes a point in $\partial H_{\mathbb{C}}^{n}$ or a point-pair $\{x, y\} \subset \partial H_{\mathbb{C}}^{n}$;

(3) $G$ is purely elliptic, i.e. each non-trivial element of $G$ is elliptic, then $G$ is elementary and fixes a point in $\overline{H_{\mathbb{C}}^{n}}$.

LEMma 2.3. (cf. [10]). Let $G$ be a discrete subgroup of $U(1, n ; \mathbb{C})$ such that every element has finite order, then $G$ is finite. 
Lemma 2.4. Let $f \in U(1, n ; \mathbb{C})$ be an elliptic element of order $m$. If $2 \leq m<M$, then there is a constant $\delta(M)$ such that

$$
N(f)>\delta(M)
$$

Proof. Let the eigenvalues of $f$ be $\lambda_{j}=e^{i \theta_{j}}(j=1, \ldots, n+1)$. By Schur's unitary triangularization theorem, there is a matrix $U \in U(n+1 ; \mathbb{C})$ such that

$$
U f \bar{U}^{T}=\left(\begin{array}{cccc}
\lambda_{1} & * & * & * \\
0 & \lambda_{2} & * & * \\
\vdots & \ddots & \ddots & * \\
0 & \cdots & 0 & \lambda_{n+1}
\end{array}\right) .
$$

Hence, $\left\|f-I_{n+1}\right\|^{2} \geq \sum_{j=1}^{n+1}\left|\lambda_{j}-1\right|^{2}=2(n+1)-2 \sum_{j=1}^{n+1} \cos \theta_{j}$. It follows from $f^{m}=$ $I_{n+1}$ that there is a $j$ such that $\left|\cos \theta_{j}\right| \neq 1$ and $\theta_{j}=\frac{2 p \pi}{m}$ (here $p$ and $m$ are prime). Hence,

$$
1-\cos \theta_{j} \geq 1-\left|\cos \theta_{j}\right|>1-\left|\cos \frac{\pi}{m}\right| \text {. }
$$

Set $\delta(M)=\sqrt{1-\left|\cos \frac{\pi}{m}\right|}$. Then $\delta(M)$ is the desired number.

From Lemma 2.4, we have the following.

COROLlaRY 2.1. If $f_{j} \rightarrow I_{n+1}$ as $j \rightarrow \infty$ and $f_{j}$ are elliptic elements with $\operatorname{ord}\left(f_{j}\right)<m$, then for all large enough $j, f_{j}=I_{n+1}$.

COROLlaRY 2.2. If $f_{j}$ are elliptic elements with ord $\left(f_{j}\right) \leq M$ and $f_{j} \rightarrow f$ as $j \rightarrow \infty$, then $f$ is an elliptic element with order $m(2 \leq m \leq M)$, and for all large enough $j$, $\operatorname{order}\left(f_{j}\right)=m$.

Proof. By the Pigeonhole Principle, we can choose a subsequence $f_{j k}$ such that each element with order $m(2 \leq m \leq M)$. Then $f_{j k}^{m} \rightarrow f^{m}$, i.e. $f$ is an elliptic element with order $m$.

Lemma 2.5. (cf. [9, Lemma 2.8]). Let $x$ and $y$ be two distinct points in $\overline{H_{\mathbb{C}}^{n}}$. If $f \in U(1, n ; \mathbb{C})$ interchanges $x$ and $y$, then

$$
N(f) \geq \sqrt{2}
$$

Proof. Since $f$ interchanges $x, y \in \overline{H_{\mathbb{C}}^{n}}$, we can find $\lambda_{i} \neq 0(i=1,2)$ such that

$$
f\left(\begin{array}{l}
1 \\
x
\end{array}\right)=\lambda_{1}\left(\begin{array}{l}
1 \\
y
\end{array}\right), f\left(\begin{array}{l}
1 \\
y
\end{array}\right)=\lambda_{2}\left(\begin{array}{l}
1 \\
x
\end{array}\right) .
$$

By the linear algebra theory, we can find $U \in U(n+1 ; \mathbb{C})$ such that

$$
U\left(\begin{array}{l}
1 \\
x
\end{array}\right)=\left(\begin{array}{l}
t_{1} \\
0
\end{array}\right), \quad U\left(\begin{array}{l}
1 \\
y
\end{array}\right)=\left(\begin{array}{l}
c_{1} \\
c_{2} \\
0
\end{array}\right)
$$

where $t_{1}, c_{1}, c_{2} \in \mathbb{C}$ and $\left|t_{1}\right|^{2}=1+\|x\|^{2},\left|c_{1}\right|^{2}+\left|c_{2}\right|^{2}=1+\|y\|^{2}$. Since $x \neq y$, we have that $c_{2} \neq 0$. 
Let $g=\left(b_{i j}\right)_{i, j=1, \ldots, n+1}=U f \bar{U}^{T}$. It follows from (2.1) and (2.2) that

$$
b_{11} t_{1}=c_{1} \lambda_{1}, b_{21} t_{1}=c_{2} \lambda_{1} \quad \text { and } \quad b_{21} c_{1}+b_{22} c_{2}=0,
$$

which implies that $b_{11}=-b_{22}$.

Thus,

$$
\begin{gathered}
\left\|f-I_{n+1}\right\|^{2}=\left\|g-I_{n+1}\right\|^{2} \geq\left|b_{11}-1\right|^{2}+\left|b_{22}-1\right|^{2} \\
\geq \frac{1}{2}\left|b_{11}+b_{22}-2\right|^{2}=2,
\end{gathered}
$$

i.e. $N(f) \geq \sqrt{2}$.

LEMMA 2.6. Let $\left\{f_{i}\right\}$ and $\left\{g_{i}\right\}$ be two sequences of $U(1, n ; \mathbb{C})$, which converge to $f$ and $g$, respectively. Suppose that each group $\left\langle f_{i}, g_{i}\right\rangle$ is a complex Kleinian group and each $f_{i}$ is of infinite order. Then $f$ is of infinite order and $\langle f, g\rangle$ is a complex Kleinian group if $\left\{\left\langle f_{i}, g_{i}\right\rangle\right\}$ satisfies I-condition.

Proof. We first prove that $\langle f, g\rangle$ is discrete.

Suppose that $\langle f, g\rangle$ is not discrete. Then there is a sequence $\left\{h_{j}\right\}$ of $\langle f, g\rangle$ such that $h_{j} \rightarrow I_{n+1}$ as $j \rightarrow \infty$. Let $h_{j, i}$ be the corresponding elements in $\left\langle f_{i}, g_{i}\right\rangle$ such that

$$
h_{j, i} \rightarrow h_{j} \text { as } i \rightarrow \infty \text {. }
$$

These elements form a sequence $h_{j_{k}, i_{k}} \in\left\langle f_{i_{k}}, g_{i_{k}}\right\rangle$ satisfying

$$
h_{j_{k}, i_{k}} \rightarrow I_{n+1} \text { as } k \rightarrow \infty .
$$

Since $\left\langle f_{i_{k}}, g_{i_{k}}\right\rangle$ is a complex Kleinian and $\left\{\left\langle f_{i}, g_{i}\right\rangle\right\}$ satisfies I-condition, by Lemma 2.4, we conclude that $h_{j_{k}, i_{k}}$ is parabolic or loxodromic.

Let $q_{1, i_{k}}$ and $q_{2, i_{k}}$ be two loxodromic elements of $\left\langle f_{i_{k}}, g_{i_{k}}\right\rangle$ having no common fixed point. Since $h_{j_{k}, i_{k}} \rightarrow I_{n+1}$, there is a positive $M$ such that for all $k>M$,

$$
\max \left\{N\left(h_{j_{k}, i_{k}}\right), N\left(\left[h_{j_{k}, i_{k}}, q_{t, i_{k}}\right]\right)\right\}<2-\sqrt{3}, \quad(t=1,2) .
$$

By Lemma 2.1 and the discreteness of $\left\langle q_{t, i_{k}}, h_{j_{k}, i_{k}}\right\rangle$, we know that $\left\langle q_{t, i_{k}}, h_{j_{k}, i_{k}}\right\rangle$ is elementary, which is a contradiction to Lemma 2.2. The above shows that $\langle f, g\rangle$ is discrete.

We now come to prove that $\langle f, g\rangle$ is non-elementary.

We first show that $f$ is parabolic or loxodromic. Since $\langle f, g\rangle$ is discrete, $f$ cannot be an irrational rotation. Suppose that there is a positive $M$ such that $f^{M}=I_{n+1}$. Then $f_{i}^{M} \neq I_{n+1}$ and

$$
f_{i}^{M} \rightarrow I_{n+1} \text { as } i \rightarrow \infty
$$

Hence, for sufficiently large $i$,

$$
\max \left\{N\left(f_{i}^{M}\right), N\left(\left[f_{i}^{M}, g_{i}^{t}\right]\right) \mid t=1,2, \ldots, n+1\right\}<2-\sqrt{3} .
$$

By Lemma 2.1, $\left\langle f_{i}^{M}, g_{i}\right\rangle$, which are subgroups of discrete group $\left\langle f_{i}, g_{i}\right\rangle$, are elementary for sufficiently large $i$. This implies that $\left\langle f_{i}, g_{i}\right\rangle$ is elementary. This is a contradiction.

We then show that $\langle f, g\rangle$ is non-elementary. 
Suppose that $\langle f, g\rangle$ is elementary. As in [9, Proposition 2.7], we can show that $\langle f, g\rangle$ is virtually abelian. Thus, there exist two integers $t$ and $s$ such that

$$
\left[f^{t}, g f^{s} g^{-1}\right]=I_{n+1} \text {. }
$$

Let $h_{i}=\left[f_{i}^{t}, g_{i} f_{i}^{s} g_{i}^{-1}\right]$. Then,

$$
h_{i} \in\left\langle f_{i}, g_{i}\right\rangle, \quad h_{i} \neq I_{n+1} \text { and } h_{i} \rightarrow I_{n+1} \text { as } i \rightarrow \infty .
$$

As in the proof of discreteness of $\langle f, g\rangle$, we can get a contradiction. Thus, $\langle f, g\rangle$ is non-elementary.

\section{Proofs of convergence theorems.}

Proof of Theorem 1.1. Assume that $G_{r}$ is non-elementary and $W\left(G_{r}\right)$ is finite. We need to prove that $G_{r}$ is discrete.

Suppose that $G_{r}$ is not discrete. Then there is a sequence $\left\{g_{j}\right\}$ of $G_{r}$ such that

$$
g_{j} \rightarrow I_{n+1} \text { as } j \rightarrow \infty
$$

We will get a contradiction by showing that each $g_{j}$ belongs to $W\left(G_{r}\right)$ for large enough $j$.

Since $G_{r}$ is non-elementary, $G_{r}$ contains two loxodromic elements $f_{1}$ and $f_{2}$ sharing no common fixed point. Then for large enough $j$,

$$
N\left(g_{j}\right)+\sum_{k=1}^{n+1} N\left(\left[g_{j}, f_{m}^{k}\right]\right)<2-\sqrt{3}(m=1,2) .
$$

Let $g_{j, t}$ and $f_{m, t}$ be the corresponding entries in $G_{r, t}$. That is $g_{j, t} \rightarrow g_{j}$ and $f_{m, t} \rightarrow f_{m}$ as $t \rightarrow \infty$. Then for large enough $t$ and $j$,

$$
N\left(g_{j, t}\right)+\sum_{k=1}^{n+1} N\left(\left[g_{j, t}, f_{m, t}^{k}\right]\right)<2-\sqrt{3}(m=1,2) .
$$

Lemma 2.1 implies that $\left\langle f_{m, t}, g_{j, t}\right\rangle(m=1,2)$ are elementary for large enough $t$ and $j$. Since $f_{m, t}$ is a loxodromic element and $g_{j, t}$ cannot interchange the two fixed points of $f_{m, t}$ for large enough $t$ and $j$, it follows from Lemma 2.2 that $f i x\left(f_{m, t}\right) \subset f i x\left(g_{j, t}\right)$ holds for each $m=1,2$ and sufficiently large $t$ and $j$. Hence, there is an integer $k_{1}$ such that for all $j \geq k_{1}, f i x\left(f_{m}\right) \subset f i x\left(g_{j}\right)$ holds for each $m=1,2$.

Let $T\left(k_{1}\right)=\bigcap_{j \geq k_{1}} f i x\left(g_{j}\right)$. Then $T\left(k_{1}\right)$ contains the linear span of fixed points of $f_{m}$ and so has dimension of at least 1 for large positive integer $k_{1}$. Thus, by passing to a subsequence of $\left\{g_{j}\right\}$ (denoted by the same manner), we have

$$
T\left(k_{1}\right) \neq \varnothing \text { and } 1 \leq \operatorname{dim}\left[T\left(k_{1}\right)\right] \leq n-1 .
$$

Suppose that there exists a loxodromic element $h \in G_{r}$ such that

$$
f i x(h) \cap T\left(k_{1}\right)=\emptyset .
$$


As an above reasoning (if needed, passing to a subsequence), there exists $k_{2}\left(>k_{1}\right)$ such that

$$
f i x(h) \subset T\left(k_{2}\right) \text { and } \operatorname{dim}\left[T\left(k_{1}\right)\right]+1 \leq \operatorname{dim}\left[T\left(k_{2}\right)\right] \leq n-1 .
$$

By repeating this step finite times, we can find $k$ such that

$$
f i x(g) \subset T(k)
$$

holds for any loxodromic element $g \in G_{r}$. Then $g_{j} \in W\left(G_{r}\right)$ for all $j>k$. This is a contradiction to the fact that $W\left(G_{r}\right)$ is finite.

The proof is complete.

Proof of Theorem 1.2. We divide our proof into three parts.

(1) First we prove that $G_{r}$ is discrete.

Suppose that $G_{r}$ is not discrete. Then there is a sequence $\left\{g_{j}\right\}$ of $G_{r}$ such that

$$
g_{j} \rightarrow I_{n+1} \text { as } j \rightarrow \infty
$$

and we can find a corresponding sequence $\left\{g_{j_{k}, i_{k}}\right\}$ such that

$$
g_{j_{k}, i_{k}} \in G_{r, i_{k}} \text { and } g_{j_{k}, i_{k}} \rightarrow I_{n+1} \text { as } k \rightarrow \infty \text {. }
$$

Since $\left\{G_{r, i}\right\}$ satisfies $I P$-condition and $G_{r, i}$ is discrete for each $i$, we may assume that for each $k, g_{j_{k}, i_{k}}$ is parabolic or loxodromic. For each $k$, there is at least one generator of $G_{r, i_{k}}$, say $f_{1, i_{k}}$, such that $\left\langle f_{1, i_{k}}, g_{j_{k}, i_{k}}\right\rangle$ is non-elementary, which is a contradiction to Lemma 2.1. The above proves the discreteness of $G_{r}$.

(2) Then we prove that $G_{r}$ is infinite.

Suppose that $G_{r}$ is finite. As in the proof of part (1) in [9, Proposition 5.8], we can find a sequence $\left\{h_{i}\right\}$ such that $\left\{h_{i}\right\} \in G_{r, i}$ and $h_{i} \rightarrow I_{n+1}$ as $i \rightarrow \infty$. Similar discussions as in the proof of part (1) show that this is impossible. Hence, $G_{r}$ is infinite.

(3) We prove that $G_{r}$ is non-elementary.

Suppose that $G_{r}$ is elementary. It follows from the infiniteness of $G_{r}$ and Lemma 2.3 that $G_{r}$ contains some element $h$ of infinite order, i.e. $h$ is parabolic or loxodromic. Let $\left\{h_{i}\right\}$ be the corresponding elements in $\left\{G_{r, i}\right\}$. Then

$$
h_{i} \rightarrow h \text { as } i \rightarrow \infty
$$

Suppose that $h$ is loxodromic. Then $h_{i}$ is loxodromic for all sufficiently large $i$. For each generator $f_{s}(s=1,2, \ldots, r)$ of $G_{r}$, as $\left\langle f_{s}, h\right\rangle$ is discrete and elementary, there exist $k_{s}$ and $p_{s}$ such that $\left[h^{k_{s}}, f_{s} h^{p_{s}} f_{s}^{-1}\right]=I_{n+1}$. Since $\left\{G_{r, i}\right\}$ satisfies $I P$-condition and $G_{r, i}$ is discrete, we have

$$
\left[h_{i}^{k_{s}}, f_{s, i} h_{i}^{p_{s}} f_{s, i}^{-1}\right] \neq I_{n+1} \text { and }\left[h_{i}^{k_{s}}, f_{s, i} h_{i}^{p_{s}} f_{s, i}^{-1}\right] \rightarrow I_{n+1} \text { as } i \rightarrow \infty .
$$

Let $g_{i}=\left[h_{i}^{k_{s}}, f_{s, i} h_{i}^{p_{s}} f_{s, i}^{-1}\right] \rightarrow I_{n+1}$ as $i \rightarrow \infty$. Then, as in the proof of part (1), we get a contradiction.

Thus, we may assume that $h$ is parabolic. Since $\left\{G_{r, i}\right\}$ satisfies $I P$-condition, by Corollary 2.2, we know that $h_{i}$ is parabolic or loxodromic.

Suppose that there is a subsequence of $\left\{h_{i}\right\}$ such that each $h_{i}$ is parabolic or loxodromic. Then for $i$, there is a generator, say $f_{1, i}$, such that the group $\left\langle f_{1, i}, h_{i}\right\rangle$ is 
non-elementary. By Lemma 2.6, the limit group of the sequence $\left\{\left\langle f_{1, i}, h_{i}\right\rangle\right\}$ is nonelementary. This implies that $G_{r}$ is non-elementary. This is a contradiction.

The proof is complete.

ACKNOWLEDGMENT. The author would like to express much gratitude to the referee for careful reading of this paper as well as many useful suggestions.

\section{REFERENCES}

1. B. N. Apanasov, Conformal geometry of discrete groups and manifolds (Walter de Gruyter, Berlin, Germany, 2000).

2. W. Cao, Discrete and dense subgroups acting on complex hyperbolic space, Bull. Aust. Math. Soc. 82 (2008), 211-224.

3. W. Cao and X. Wang, Discreteness criteria and algebraic convergence theorem for subgroups in PU(1, $n ; \mathbb{C})$, Proc. Japan Acad. 82 (2006), 49-52.

4. S. Chen and L. Greenberg, Hyperbolic spaces, in Contributions to analysis (Academic Press, New York, 1974), 49-87.

5. B. Dai, A. Fang and B. Nai, Discreteness criteria for subgroups in complex hyperbolic space, Proc. Japan Acad. 77 (2001), 168-172. 1999).

6. W. M. Goldman, Complex hyperbolic geometry (Oxford University Press, New York,

7. T. Jørgensen and P. Klein, Algebraic convergence of finitely generated Kleinian groups, Quart. J. Math. 33 (1982), 325-332.

8. S. Kamiya, Notes on elements of $U(1, n ; \mathbb{C})$, Hiroshima Math. J. 21 (1991), $23-45$.

9. G. J. Martin, On discrete Möbius groups in all dimensions, Acta Math. 163 (1989), 253-289.

10. J. P. Navarrete, On the limit set of discrete subgroups of $\mathrm{PU}(2,1)$, Geometriae Dedicata 122 (2006), 1-13.

11. X. Wang, Algebraic convergence theorems of n-dimensional Kleinian groups, Isr. $J$. Math. 162 (2007), 221-233.

12. X. Wang and W. Yang, Discreteness criteria of Möbius groups of high dimensions and convergence theorem of Kleinian groups, Adv. Math. 159 (2001), 68-82. 